\title{
Altered macrophage differentiation and immune dysfunction in tumor development
}

\author{
Antonio Sica ${ }^{1}$ and Vincenzo Bronte ${ }^{2}$ \\ ${ }^{1}$ Istituto Clinico Humanitas, Instituto di Ricovero e Cura a Carattere Scientifico (IRCCS), Rozzano, Italy. ${ }^{2}$ Istituto Oncologico Veneto, IRCCS, Padua, Italy.
}

\begin{abstract}
Tumors require a constant influx of myelomonocytic cells to support the angiogenesis and stroma remodeling needed for their growth. This is mediated by tumor-derived factors, which cause sustained myelopoiesis and the accumulation and functional differentiation of myelomonocytic cells, most of which are macrophages, at the tumor site. An important side effect of the accumulation and functional differentiation of these cells is that they can induce lymphocyte dysfunction. A complete understanding of the complex interplay between neoplastic and myelomonocytic cells might offer novel targets for therapeutic intervention aimed at depriving tumor cells of important growth support and enhancing the antitumor immune response.
\end{abstract}

Although clinical trials evaluating the effectiveness of novel cancer vaccines indicate that in cancer patients they can induce robust immune responses against tumor antigens, the clinical benefits of these vaccines have been limited $(1,2)$. The reasons behind these limited clinical responses are not known but might be related, in part, to the immunosuppressive effects of tumors. Immune dysregulation and suppression in cancer patients is a composite event in which tumor-derived factors condition not only peripheral immune niches, in which dysfunction and even death of tumor-specific $T$ cells can occur, but also the bone marrow and other hematopoietic organs (such as the mouse spleen), leading to abnormal myelopoiesis and the accumulation of immunosuppressive myelomonocytic cells at the tumor site $(3,4)$. Dysregulation and/or suppression of tumor-specific $\mathrm{T}$ cell function(s) is therefore likely to occur at 2 separate sites: locally, at the tumor-host interface, where cancer cells directly condition the tumor stroma; and systemically, where an expanded pool of immature and immunosuppressive myeloid cells are free to circulate and mediate suppression in the blood and lymphoid organs. This Review attempts to analyze the main myeloid cell populations that restrain antitumor immune responses.

\section{Immunosuppression and cancer: history and nomenclature}

Although a population of not very well defined cells called natural suppressors was associated in the early 1980s with immune suppression and tumor development (5), the first description indicating that increased numbers of myeloid cells in tumor-bearing hosts might alter antitumor immune reactivity was provided by Hans Schreiber's group $(6,7)$. In one key experiment, the administration of a Gr-1-specific antibody that recognizes both Ly6C and Ly6G to immunocompetent mice reduced the growth of a variant of a UV light-induced tumor able to progress more aggressively than its parental tumor cell line (6). This variant was known

Nonstandard abbreviations used: ARG1, arginase 1; CCL2, CC chemokine ligand 2; $\mathrm{CD} 3 \zeta, \zeta$ chain of the CD3 component of the TCR complex; CXCL12, CXC chemokine ligand 12; CXCR4, CXC chemokine receptor 4; HIF-1, hypoxia-inducible factor 1; IL-4R $\alpha$, IL-4 receptor $\alpha$-chain; MDSC, myeloid-derived suppressor cell; SHIP, Src homology 2 domain-containing inositol-5-phosphatase; TAM, tumor-associated macrophage.

Conflict of interest: The authors have declared that no conflict of interest exists. Citation for this article: J. Clin. Invest. 117:1155-1166 (2007). doi:10.1172/JCI31422. to attract more leukocytes than the parental cell line, a property attributed to the release of a noncharacterized chemotactic factor, and its growth in vivo was known to be restrained mainly by $\mathrm{CD}^{+}$ $\mathrm{T}$ cells. Interestingly, elimination of $\mathrm{Gr}-1^{+}$cells in athymic nude mice (which lack most $\mathrm{T}$ cells) also slowed the growth of this aggressive variant, suggesting that $\mathrm{Gr}-1^{+}$leukocytes in tumor-bearing hosts might also promote tumor growth and development (7). The effect of in vivo treatment with this Gr-1-specific antibody was originally attributed to the elimination of granulocytes (which are known to express high levels of Ly6G but low levels of Ly6C), but successive reports from several groups indicated that the Gr-1-specific antibody could bind and eliminate other cells in the blood. Gr- $1^{+}$cells in tumor-bearing hosts were, in fact, mostly CD11 $\mathrm{b}^{+}$and comprised both polymorphonuclear and mononuclear cells, including cells at different stages of maturation along the myelomonocytic differentiation pathway, thereby revealing a profound alteration in myelopoiesis during tumor progression $(4,8)$ (Figure 1). Myelopoiesis, in fact, is not only increased in the bone marrow and spleen of tumor-bearing mice but is also altered, since the myelomonocytic cells cannot properly differentiate into professional APCs, such as DCs (reviewed in ref. 9).

\section{Heterogeneity of myeloid-derived suppressor cells}

The heterogeneity of the $\mathrm{CD} 11 \mathrm{~b}^{+} \mathrm{Gr}-1^{+}$cells has generated some confusion, in particular because of the nomenclature used previously to define them (i.e., immature myeloid cells or myeloid suppressor cells). Recently, a panel of leading investigators in the field agreed to use the common term myeloid-derived suppressor cells (MDSCs) (10). The MDSC definition involves a synthesis of the functional and phenotypic properties of the cells. MDSCs can be defined as a population of myelomonocytic cells normally lacking the markers of mature myeloid cells and commonly expressing both Gr-1 and CD11b in mice, with a high potential to suppress immune responses in vitro and in vivo. The exact nature of the MDSC population depends on various factors described below, the most important of which is probably the tumor type.

Even though numerous findings suggest that the monocytic, rather than the granulocytic, fraction of mouse $\mathrm{CD} 11 \mathrm{~b}^{+} \mathrm{Gr}-1^{+}$cells is responsible for the immune dysfunctions induced by this cell population, both in vitro and in vivo, in antigen-specific $\mathrm{CD}^{+}$ $\mathrm{T}$ cells (11-13), the use of the term myeloid is justified by the incomplete understanding of the relationship between the two 


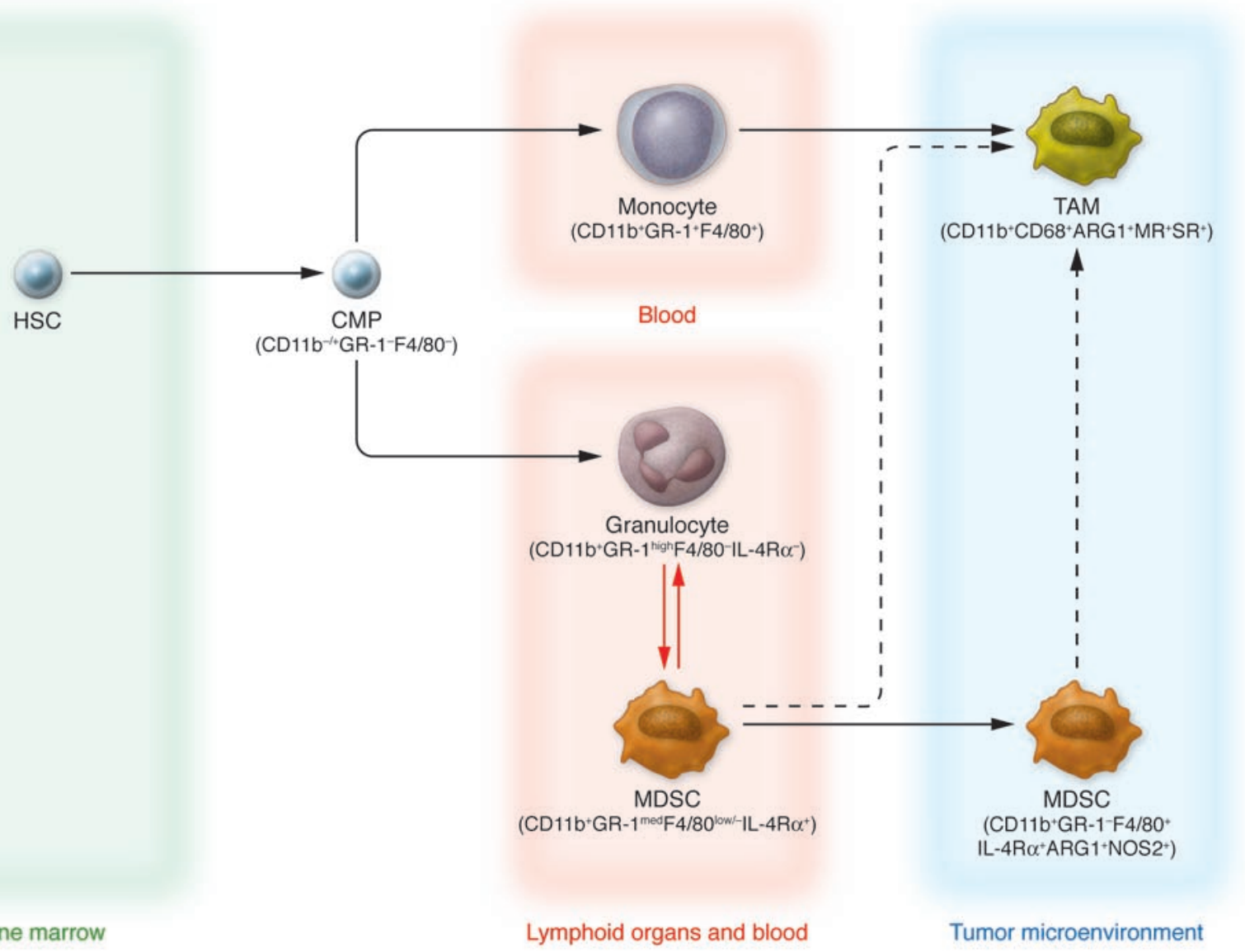

Figure 1

Current view of TAM and MDSC differentiation. HSCs give rise to common myeloid precursors (CMPs), which subsequently originate at least three subsets of cells circulating in tumor-bearing hosts that can be identified by specific markers: monocytes $\left(\mathrm{CD} 11 \mathrm{~b}^{+} \mathrm{Gr}-1^{+} \mathrm{F} 4 / 80^{+}\right)$, granulocytes

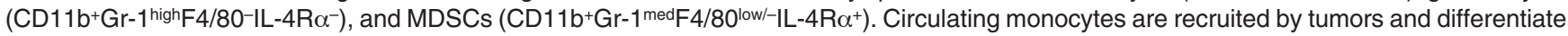
into TAMs, acquiring protumoral functions. During tumor progression, MDSCs accumulating in blood and in lymphoid organs such as the spleen may also be recruited to the tumor microenvironment, where they become F4/80+. This latter pathway of MDSC-TAM phenotype transition (dashed arrow) was recently proposed $(13,27)$. Finally, it has been hypothesized that immature forms of granulocytes might differentiate into MDSCs or condition their function and/or further differentiation (red arrows), as suggested by some studies (14).

main progeny of the enhanced myelopoiesis observed in tumorbearing hosts (i.e., granulocytes and monocytes; Figure 1). Both MDSCs and tumor-associated macrophages (TAMs) have a phenotype similar to that of alternatively activated macrophages (also known as M2 macrophages) in the mouse, as discussed below, and tumor-conditioned granulocytes might have a role in influencing this activation process. It must be pointed out, in fact, that in mice, three different neutrophil subsets have been isolated that can condition monocyte/macrophage differentiation toward the classic or alternative activation pathway by releasing different cytokines and chemokines (14). Furthermore, human granulocyte subpopulations in patients with renal cell cancer have been shown to function as $\operatorname{MDSCs}(15,16)$.

$\mathrm{CD} 11 \mathrm{~b}+\mathrm{Gr}-1^{+}$cells are normally present in the bone marrow of healthy mice and accumulate in the spleen and blood of tumorbearing mice $(17-20)$. CD $11 b^{+} \mathrm{Gr}-1^{+}$cells present in steady-state conditions are not able to induce suppression of antigen-stimulated $\mathrm{T}$ cells, at least not to the same extent as the cells that accumulate in tumor-bearing mice, and recent data support the possibility that exogenously provided IL-13 might confer on them suppressive activity $(21,22)$. Berzofsky and colleagues have shown that a subset of NKT cells recognizing tumor-derived glycolipids presented by the MHC-like molecule CD1 releases IL-13. This IL-13 can then activate $\mathrm{CD} 11 \mathrm{~b}^{+} \mathrm{GR}-1^{+}$cells to suppress tumor-specific CTLs through a STAT 6 pathway initiated by the IL- 4 receptor $\alpha$-chain (IL-4R $\alpha$ ), which is common to the receptors for IL-4 and IL-13 (21, 22). This circuit is activated very early after tumor implantation in mice, before any increase in the number of CD $11 b^{+} \mathrm{Gr}-1^{+}$cells is detected. In several experimental models, however, systemic accumulation of $\mathrm{CD} 11 \mathrm{~b}^{+} \mathrm{Gr}-1^{+}$cells, probably resulting from both differentiation of precursors and recruitment to particular anatomical sites, precedes and is important for mediating suppression of $\mathrm{T}$ cells, not only in cancer but also during infections (Table 1).

\section{MDSC suppression of T cell function}

The biology and properties of MDSCs in tumor-bearing hosts have been extensively described in recent reviews $(4,8,23)$ and are summarized here in Properties of MDSCs. The mechanisms underlying 


\section{Table 1}

Myeloid cell-dependent suppression of T cells in mice

\begin{tabular}{|c|c|c|c|c|c|}
\hline Pathology & $\begin{array}{l}\text { Suppressor cells } \\
\text { isolated from: }\end{array}$ & Phenotype & Mouse strain & Mechanism of T cell inhibition & References \\
\hline \multicolumn{6}{|l|}{ Cancer } \\
\hline $\begin{array}{l}\text { Colon carcinomas } \\
\text { (CT26 and C26) }\end{array}$ & Spleen and tumor & $\mathrm{CD} 11 \mathrm{~b}+\mathrm{Gr}-1^{+}$ & $\mathrm{BALB} / \mathrm{c}$ & ARG and NO dependent & $\begin{array}{l}(11,17 \\
44,115)\end{array}$ \\
\hline Melanoma (B16) & Spleen & $\mathrm{CD} 11 \mathrm{~b}+\mathrm{Gr}-1^{+}$ & $\mathrm{C} 57 \mathrm{BL} / 6$ & NOS dependent & $(45)$ \\
\hline Lymphoma (EL-4) & Tumor & $\mathrm{CD} 11 \mathrm{~b}+\mathrm{Gr}-1+\mathrm{F} 4 / 80^{+}$ & C57BL/6 & ARG and NO dependent & (13) \\
\hline $\begin{array}{l}\text { Colon adenocarcinoma } \\
\text { (MCA-38) }\end{array}$ & Tumor & $\mathrm{F} 4 / 80^{+}$ & C57BL/6 & $\begin{array}{l}\text { NO and cell-associated form } \\
\text { of TNF- } \alpha\end{array}$ & $(116)$ \\
\hline Mammary carcinoma (4T1) & Spleen & $\mathrm{CD} 11 \mathrm{~b}+\mathrm{Gr}-1+\mathrm{CD} 11 \mathrm{c}^{+}$ & $\mathrm{BALB} / \mathrm{c}$ & ARG dependent & $(20)$ \\
\hline Lewis lung carcinoma & Tumor & $\mathrm{CD} 11 \mathrm{~b}+\mathrm{Gr}-1-\mathrm{F} 4 / 80^{-} \mathrm{CD} 80^{+}$ & $\mathrm{C} 57 \mathrm{BL} / 6$ & ARG dependent & $(47)$ \\
\hline Lewis lung carcinoma & Tumor & $\mathrm{CD} 31^{+}$ & $\mathrm{C} 57 \mathrm{BL} / 6$ & NO and TGF- $\beta$ dependent & $(117)$ \\
\hline T cell lymphoma (BW-Sp3) & Spleen & CD11b+Gr-1 int Ly6G-CD115 int & AKR & $\begin{array}{l}\text { ARG and NO independent; } \\
\text { partially dependent on PPAR } \gamma\end{array}$ & $(12,118)$ \\
\hline Fibrosarcoma (C3) & Spleen & $\mathrm{CD} 11 \mathrm{~b}+\mathrm{Gr}-1^{+}$ & $\mathrm{C} 57 \mathrm{BL} / 6$ & ARG and $\mathrm{H}_{2} \mathrm{O}_{2}$ & (19) \\
\hline $\begin{array}{l}\text { Transformed fibroblasts } \\
\quad(15-12 \mathrm{RM})\end{array}$ & Spleen & $\mathrm{CD} 11 \mathrm{~b}+\mathrm{Gr}-1^{+}$ & $\mathrm{BALB} / \mathrm{c}$ & $\begin{array}{l}\text { NKT cells, IL-13, STAT6, TGF- } \beta \text {; } \\
\text { NOS independent; ARG not tested }\end{array}$ & $(21,22)$ \\
\hline \multicolumn{6}{|l|}{ Infection } \\
\hline Candida albicans & Blood, spleen & $\begin{array}{c}\mathrm{CD} 11 \mathrm{~b}+\mathrm{Gr}-1+\mathrm{CD} 80^{+} \\
\text {polymorphonuclear cells }\end{array}$ & $\mathrm{BALB} / \mathrm{c}$ & IFN- $\gamma / \mathrm{NO}$ and CD80 & (119) \\
\hline Trypanosoma cruzi & Spleen & $\mathrm{CD} 11 \mathrm{~b}+\mathrm{Gr}-1^{+}$ & C57BL/6 and Sv129 & IFN- $\gamma /$ NOS & $(120)$ \\
\hline Schistosoma mansoni & Spleen & $\mathrm{CD} 11 \mathrm{~b}+\mathrm{Gr}-1+\mathrm{CD} 16+$ & BALB/C and B10.D2 & $\begin{array}{l}\text { Unidentified soluble factor } \\
\text { (not IL-4, IL-10, or TGF- } \beta \text { ) }\end{array}$ & (121) \\
\hline Taenia crassiceps & Peritoneum & $\mathrm{CD} 11 \mathrm{~b}+\mathrm{Gr}-1^{+}$ & $\mathrm{BALB} / \mathrm{c}$ & 12/15-Lipoxygenase, NO and ARG & $(122)$ \\
\hline $\begin{array}{l}\text { Porphyromonas } \\
\text { gingivalis }\end{array}$ & $\begin{array}{l}\text { Spleen, BM but } \\
\text { not lymph nodes }\end{array}$ & $\mathrm{CD} 11 \mathrm{~b}+\mathrm{Gr}-1^{+}$ & $\mathrm{BALB} / \mathrm{C}$ & $\mathrm{IFN}-\gamma$ & $(52)$ \\
\hline $\begin{array}{l}\text { Schistosome } \\
\text { oligosaccharide } \\
\text { (Lacto- } N \text {-neotetraose) }\end{array}$ & Peritoneum & $\mathrm{CD} 11 \mathrm{~b}+\mathrm{Gr}-1+\mathrm{F} 4 / 80^{+}$ & $\mathrm{BALB} / \mathrm{C}$ and $\mathrm{C} 57 \mathrm{BL} / 6$ & IFN- $\gamma / N 0$; partly IL-10 dependent & (123) \\
\hline $\begin{array}{l}\text { Cruzipain antigen } \\
\quad \text { from T. cruzi }\end{array}$ & $\begin{array}{c}\text { Spleen } \\
\text { (extramedullary } \\
\text { hematopoiesis) }\end{array}$ & $\mathrm{CD} 11 \mathrm{~b}+\mathrm{Gr}-1^{+}$ & $\mathrm{BALB} / \mathrm{C}$ & $\begin{array}{l}\text { Not investigated; ARG and NOS } \\
\text { activity increased in macrophages }\end{array}$ & (124) \\
\hline
\end{tabular}

the inhibitory activity of MDSCs are probably various, ranging from those requiring direct cell-cell contact to others indirectly mediated by modification of the microenvironment. MDSCs freshly isolated from the spleens of tumor-bearing mice were originally shown to suppress the functional activity of $\mathrm{CD}^{+} \mathrm{T}$ cells, but not $\mathrm{CD}^{+} \mathrm{T}$ cells, by interfering with their ability to secrete IFN- $\gamma$ when stimulated with specific antigens $(19,24)$. This effect was thought to be related to the fact that MDSCs expressed MHC class I but not MHC class II and was mediated by cell-cell contact and the production of ROS such as hydrogen peroxide $\left(\mathrm{H}_{2} \mathrm{O}_{2}\right)$, triggered by MDSC expression of the enzyme arginase 1 (ARG1) (19). The role of $\mathrm{H}_{2} \mathrm{O}_{2}$ as a mediator of $\mathrm{T}$ cell dysfunction seems to correlate, at least in some studies, with decreased expression of the $\zeta$ chain of the CD3 component of the TCR complex (CD3 $\zeta$ ) (25). Other studies have shown that circulating MDSCs have to be activated by antigen-experienced $T$ cells to execute their suppressive program and that they can suppress, in an MHC-independent fashion, both antigen-activated $\mathrm{CD}^{+}{ }^{+}$and $\mathrm{CD}^{+} \mathrm{T}$ cells $(11,13,20)$. Moreover, a subset of MDSCs (expressing CD11b, Gr-1, CD115, and F4/80) isolated from the bone marrow and spleens of tumor-bearing mice can induce the development of FOXP3 ${ }^{+} \mathrm{CD}^{+}$(FOXP3, forkhead box $\mathrm{p} 3$ ) Tregs in vivo by a pathway requiring IFN- $\gamma$ and IL-10 (26). Interestingly, production of NO was not required for MDSC induction of Tregs whereas NO, released by NOS, has been shown to be extensively involved in the $\mathrm{T}$ cell dysfunction induced by MDSCs (Table 1), suggesting that the different biological activities of MDSCs might be separated at the molecular level and perhaps targeted by distinct therapeutic approaches.

Some issues must be considered when analyzing the partially conflicting results on the mechanism of MDSC-dependent suppression of $\mathrm{T}$ cells. The in vitro assays evaluating the inhibitory properties of MDSCs are not standardized, so in different studies they might differ both in the type of stimuli and source of T cells. When $\mathrm{T}$ cells are stimulated in vitro in the presence of supraphysiologic numbers of MDSCs, the mechanisms governing suppression might differ from those activated in in vitro assays where the ratio of MDSCs to $T$ cells is the same as found in the lymphoid organs of mice, where MDSCs are recruited in pathological situations. In contrast to the in vitro assays, the ability of MDSCs to induce tumor-specific $\mathrm{CD}^{+} \mathrm{T}$ cells to become nonfunctional in vivo has been repeatedly confirmed, although many studies are based on the use of either small molecules affecting MDSC inhibitory pathways or antibodies depleting $\mathrm{Gr}-1^{+}$cells (11, 22, 27-29). It must be emphasized that the interpretation of in vivo experiments with inhibitors is complicated by the possibility that these molecules affect cells other than MDSCs. 


\section{Properties of MDSCs}

Coexpression of the myeloid cell markers CD11b and Gr-1 must be associated with the functional ability to inhibit T cell activation.

Normally found in the bone marrow (in the spleen of normal mice they normally account for less than 5\% of nucleated cells), MDSCs can be increased in numbers in spleen and blood under pathological conditions. An increase in MDSC numbers in lymph nodes has been reported by some studies under pathological situations $(27,105)$.

MDSCs present at the tumor site and a fraction of cells present in the spleen of mice bearing tumors are $\mathrm{CD} 11 \mathrm{~b}^{+} \mathrm{F} 4 / 80^{+} \mathrm{Gr}-1^{-}$; these cells can arise in vivo and in vitro from $\mathrm{CD} 11 \mathrm{~b}^{+} \mathrm{Gr}-1^{+}$precursors and retain their suppressive properties $(12,13,17,28)$.

In vitro effects: MDSCs inhibit $\mathrm{T}$ cell activation $\left(\mathrm{CD}^{+} \mathrm{T}\right.$ cells more than $\mathrm{CD} 4^{+} \mathrm{T}$ cells $)$ induced by either antigens or polyclonal stimuli through an $\mathrm{MHC}$-independent mechanism requiring cell-cell contact.

Even though direct antigen presentation to the T cells by MDSCs is not required for in vitro suppression, MDSCs can take up and cross-present tumor-associated antigens in the context of MHC class I molecules in vivo (27). In this case, selective impairment of tumor-specific immunity has been shown, indicating that MHC-dependent responses might be relevant in vivo.

Human MDSC equivalents are not entirely known, but granulocyte subpopulations might be involved in mediating some human MDSC inhibitory activities $(15,16)$.

VEGF, GM-CSF, IL-3, M-CSF, and IL-6 have been shown to be involved in the alteration of normal myelopoiesis and recruitment of MDSCs to peripheral organs under pathological situations. Cytokines might be relevant for enhanced myelopoiesis, mobilization of MDSCs, and conditioning the maturation of these cells $(4,9)$.

\section{General properties of MDSCs and their relationship with M2 macrophages}

The suppressive program of MDSCs can be triggered by their interaction with antigen-activated $\mathrm{CD}^{+} \mathrm{T}$ cells both in vitro and in vivo, through an IFN- $\gamma$ - and cell-contact-dependent step that might require the expression of $\mathrm{CD} 80$ and $\mathrm{CD} 11 \mathrm{~b}$ on the surface of the MDSCs $(11,19,30)$. Interestingly, simple in vitro culture of MDSCs alone can activate this program. The reason behind the common finding that cells isolated either with Gr-1-specific or CD11bspecific antibodies and cultured in vitro (with or without GM$\mathrm{CSF}$ ) become macrophage-like cells (i.e., they gain a CD11 $\mathrm{b}^{+} \mathrm{Gr}-1^{-}$ $\mathrm{F} 4 / 80^{+} \mathrm{CD} 80^{+} \mathrm{MHC}$ class $\mathrm{II}^{-}$/low phenotype) with enhanced immunosuppressive activity (11-13) has not been fully investigated.

The inhibitory properties of MDSCs are probably mediated by the expression of inducible forms of NOS (i.e., NOS2) and ARG (i.e., ARG1). Both NOS2 and ARG1 are involved in the metabolism of the amino acid L-Arg (Figure 2). NOS2, a heme-containing enzyme that catalyzes the synthesis of NO and citrulline from L-Arg, is expressed by various cells of the immune system, and its activation is considered a hallmark of classically activated macrophages (also known as M1 macrophages), a macrophage subset that produces proinflammatory cytokines and acts as the effector cell in the killing of invading pathogens (31-33). In M1 macrophages, expression of the gene encoding NOS2 depends on the activation of transcription factors, such as NF-кB, JAK3, and STAT1 as well as JNK (34), and it can be transcriptionally upregulated by proinflammatory cytokines (e.g., IFNs, IL-1, IL-2, and TNF- $\alpha$ ), bacterial LPS, and hypoxia $(35,36)$. By contrast, ARG1 (also known as liver-type ARG because it is found predominantly in hepatocytes) is a manganese metalloenzyme that catalyzes the hydrolysis of L-Arg to L-ornithine and urea (Figure 2). However, ARG1 is also induced in cells of the innate immune system by several cytokines including TGF- $\beta$ (37), the macrophage-stimulating protein (MSP) acting on the receptor RON (38), GM-CSF (39), and either IL-4 or IL-13, both of which activate a STAT6 signaling pathway (40). In contrast to NOS2, whose activation is considered a hallmark of M1 macrophages, ARG1 activation has been regarded as one of the most specific markers of M2 macrophages, which act as important mediators of allergic responses, control parasitic infections, mediate wound repair and fibrosis, and have been found in the leukocyte infiltrates of various human and mouse tumors, where they have been suspected of promoting tumorigenesis $(31,32)$, as further discussed below. Despite this distinct expression of NOS2 and ARG1 in M1 and M2 macrophages, respectively, MDSCs have been shown to express NOS2 and/or ARG1, and recent studies indicate that MDSCs have characteristics of both M1 and M2 macrophages. Indeed, we recently described in tumor-bearing mice a population of circulating $\mathrm{CD} 11 \mathrm{~b}+\mathrm{Gr}-1^{+}$inflammatory monocytes expressing IL-4R $\alpha$ and able to release both IL-13 and IFN- $\gamma(11)$, characteristics that are compatible with a function intermediate between those of M1 and M2 macrophages. To suppress CD8 ${ }^{+}$ $\mathrm{T}$ cells, these circulating inflammatory monocytes had to be activated by IFN- $\gamma$ produced by antigen-stimulated $T$ cells, release their own IFN- $\gamma$ and IL-13, and be responsive to IL-13 by expressing a functional IL-13 receptor, including the IL-4R $\alpha$ subunit (11). IL-4R $\alpha$ is therefore a useful marker for discriminating between populations of immunosuppressive MDSCs (IL-4R $\alpha^{+}$) and nonsuppressive granulocytes (IL-4R $\alpha^{-}$), both of which are increased in the blood and spleens of tumor-bearing mice (Figure 1). Cooperation between IL-13 and IFN- $\gamma$ led to sustained activation of both ARG1 and NOS2 in MDSC populations, causing dysfunctional $\mathrm{T}$ cell responses (11). Importantly, CD11 $\mathrm{b}^{+} \mathrm{TAMs}$ also require the same combination of cytokines (IL-13 and IFN- $\gamma$ ) to mediate suppression of $\mathrm{CD}^{+} \mathrm{T}$ cells (11). These results suggest that MDSCs 


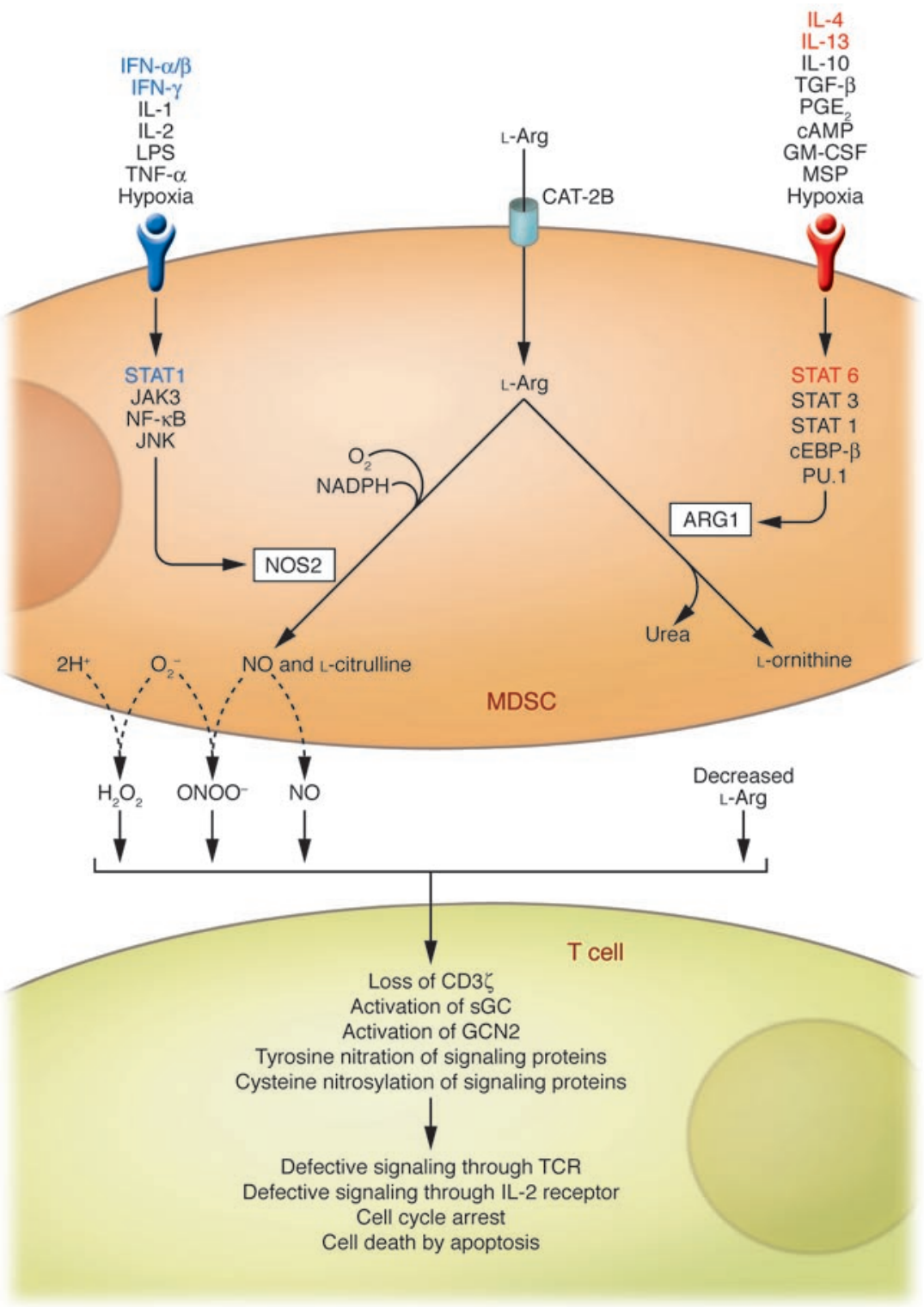

\section{Figure 2}

Inhibitory effects of MDSC L-Arg metabolism on antigen-activated T cells. L-Arg enters MDSCs through a cationic amino acid transporter (CAT-2B) and is mainly metabolized by the inducible forms of NOS and ARG (i.e., NOS2 and ARG1, respectively) although the contribution of other isoforms cannot be ruled out. Depending on the balance between these enzymes, depletion of extracellular L-Arg concentration, NO release, and enhanced production of reactive oxygen and nitrogen species (for example, $\mathrm{O}_{2}^{-}$and $\mathrm{H}_{2} \mathrm{O}_{2}$, and $\mathrm{ONOO}^{-}$, respectively) can ensue. $T$ cells that are activated in the MDSC-conditioned environment stop proliferating and eventually die by apoptosis through pathways involving activation of general control nonderepressible 2 (GCN2) and soluble guanylate cyclase (sGC); tyrosine nitration and S-cysteine nitrosylation of various proteins; loss of $\mathrm{CD} 3 \xi$; and interference with the IL-2R signaling pathway (reviewed in ref. 23). cEBP- $\beta$, CCAAT enhancer-binding protein $\beta$; MSP, macrophage-stimulating protein.

(42), and ARG1-expressing granulocytes have been reported to induce both decreased $\mathrm{CD} 3 \xi$ expression and attenuated activation in T cells from renal cell carcinoma patients (15). These discrepancies between humans and mice might reflect our incomplete understanding of the highly dynamic process of myeloid differentiation in cancer, and only the identification of the molecules released by tumors and the transcription factors activated in hematopoietic precursors can address these issues. We are currently evaluating the possibility of generating MDSCs from bone marrow precursors using defined in vitro culture systems in an attempt to address some of these issues.

\section{L-Arg metabolism as the mechanism of MDSC immunosuppression}

Increased L-Arg metabolism, either in myeloid cells infiltrating the tumor stroma or in tumor

and TAMs respond with an M2 macrophage-oriented program to classic signals driving macrophage activation (dependent on Th1 cytokines) and reconcile conflicting data attributing a prevalence of either IFN- $\gamma$, NOS2, and STAT1 or IL-4/IL-13, ARG, and STAT6 axes in the suppression of the immune response in tumor-bearing hosts (Table 1, Figure 2; also discussed further below).

Many questions, however, still await answers. It is not clear, for example, whether all the MDSC precursors in a population respond similarly (and synchronously) to $\mathrm{T}$ cell-mediated activation or whether MDSC populations are heterogeneous, with some cells programmed to activate an M1 phenotype and others to activate an M2 phenotype. Alternatively, some plasticity might exist, i.e., MDSCs might be able to oscillate between M1 and M2 phenotypes, depending upon the stimulation they receive. Moreover, with respect to the status of polarization, some differences have been reported between mice and humans. For example, ARG1 is expressed in mouse, but not human, M2 macrophages (41). In humans, ARG1 is constitutively expressed by granulocytes cells, can impair antigen responsiveness of $\mathrm{T}$ cells, both at the tumorhost interface and systemically $(23,29,43)$. Immune regulation by L-Arg metabolism is not antigen-specific, but to be susceptible to the inhibitory activity of the ARG- and NOS-dependent L-Arg metabolism pathways, a T cell must be activated through its TCR. Activation through the TCR promotes $\mathrm{T}$ cell cycling, and many of the inhibitory effects of L-Arg-metabolizing enzymes require actively proliferating cells. NOS2 and ARG1 can function separately or synergistically to alter $\mathrm{T}$ cell function; activation of either enzyme alone in an APC inhibits its ability to induce $\mathrm{T}$ cell proliferation by interfering with intracellular $\mathrm{T}$ cell signal transduction pathways whereas induction of both enzymes generates highly reactive oxygen and nitrogen species, such as $\mathrm{H}_{2} \mathrm{O}_{2}$ and peroxynitrites, that might induce signaling defects in proximal immune cells and force antigen-activated $\mathrm{T}$ cells to undergo apoptosis (Figure 2 and ref. 23). The relative levels of expression of the 2 enzymes seem to be related to the stimulus driving MDSC accumulation (Table 1). In the case of tumor-induced MDSCs, the main factors determining 


\section{Properties of TAMs}

TAMs are derived from circulating monocytes that are recruited to tumors by chemotactic factors such as CCL2, VEGF, and M-CSF $(32,106)$.

TAMs preferentially localize in hypoxic areas of tumors $(64,107)$.

M2 macrophage polarization: TAMs express high levels of M2 macrophage markers (IL-10, TGF- $\beta$, ARG1, and the mannose receptor) and low levels of mediators of M1 macrophage-mediated inflammation (IL-12, TNF- $\alpha$, and IL-6) $(32,75,81)$.

TAMs exhibit defective NF-кB activity and functional IRF-3/STAT1 pathway activity in response to TLR4 ligands (75).

TAMs exhibit the following protumoral functions:

(a) Induction of angiogenesis through expression of tissue factors, VEGF, CCL2, FGF2, CXCL8, CXCL1, and CXCL2 $(32,85,108,109)$

(b) Production of growth factors (e.g., PDGF, EGF, and VEGF) $(85,110,111)$

(c) Induction of matrix remodeling through the production of TGF- $\beta$, CCL2, and MMPs such as MMP9 $(32,112)$

(d) Immune suppression, through the production of immunosuppressive cytokines (e.g., IL-10 and TGF- $\beta$ ) $(32,75,76)$ and the recruitment of Tregs through the secretion of CCL22 (113)

(e) Skewing of adaptive immunity to a Th2-type immune response through the production of CCL17 (32), CCL18 (114), and CCL22 (113).

Increased numbers of TAMs correlate with vessel density and poor prognosis (56).

which L-Arg-metabolizing enzyme is expressed at the highest level are as follows: tumor histology, anatomical site from which the MDSCs are isolated (spleen, blood, or tumor), genetic background of mouse (which probably dictates the Th1 vs. Th2 orientation of the immune response), and type of stimulatory signal delivered to the activating $T$ cells (Table 1 and refs. 44-46).

Interestingly, as discussed above, activation of ARG1 can lead to loss of cell surface expression of $\mathrm{CD} 3 \zeta$ in antigen-activated $\mathrm{T}$ cells by consumption of $\mathrm{L}-\mathrm{Arg}$ and activation of the amino acid-deficiency sensor general control nonderepressible 2 (GCN2) $(47,48)$, a sensor that is also triggered by another amino acidmetabolizing enzyme causing immune suppression, indoleamine 2,3-dioxygenase $(49,50)$. The loss of CD3 $\zeta$ seems to be more important for inhibition of $\mathrm{CD}^{+} \mathrm{T}$ cell function than of $\mathrm{CD}^{+}$ $\mathrm{T}$ cell function (51). Indeed, splenic MDSCs were shown to induce the $\mathrm{CD} 3 \zeta$ chain downregulation in antigen-stimulated $\mathrm{CD} 4^{+}$but not $\mathrm{CD}^{+} \mathrm{T}$ cells (51). Moreover, $\mathrm{CD} 3 \zeta$ loss might not be related exclusively to tumor MDSCs, since MDSCs expanded during chronic inflammation induced by infection with Porphyromonas gingivalis can also induce its downregulation (52). It has been proposed that the functional role of MDSCs is to limit chronic stimulation of the immune response and prevent unmitigated $\mathrm{T}$ cell activation, which can be dangerous (53). Downregulation of $\mathrm{CD} 3 \zeta$ expression and the unresponsiveness of $\mathrm{T}$ cells that ensues contribute to the inflammatory response being attenuated; i.e., the release of proinflammatory cytokines and other mediators that might be detrimental to the body when produced in excess or for a prolonged period is attenuated.

Loss of CD3 $\zeta$ T cells is not the only mechanism by which heightened L-Arg metabolism mediates T cell suppression. For example, $\mathrm{CD}^{+}$tumor-infiltrating lymphocytes (TILs) present in individuals with prostate cancer are inhibited by a pathway dependent on the intratumoral activation of ARG2 and NOS2 (expressed by the cancer cells), but these TILs do not show altered expression of CD3ל or other profound defects in the TCR signaling pathway (54). We therefore think that it is probable that $\mathrm{CD} 3 \xi$ downregulation is a late event in tumor progression, associated with a deeper alteration in host myelopoiesis.

\section{Origin and molecular basis of TAM functions}

TAMs are the second well-described population of myeloid cells that have been shown to exert a negative effect on antitumor immune responses. The relationship between TAMs and MDSCs is not completely defined, but data discussed below suggest TAMs might, in part, be derived from or related to MDSCs (Figure 1 and Properties of TAMs).

For decades, solid tumors have been known to be strongly infiltrated by inflammatory leukocytes, and accumulating evidence has clearly demonstrated, in various mouse and human malignancies, including colon, breast, lung, and prostate cancer $(32,55-57)$, a strict correlation between increased numbers and/or density of macrophages and poor prognosis. Based on this, both the recruitment and activation of TAMs are regarded as pivotal to tumor progression, and TAMs are putative targets for therapeutic intervention.

As originally described by Alberto Mantovani and colleagues in the early 1980s (57), circulating monocytes (Figure 1) are recruited to the tumor, where they differentiate into TAMs, by a tumorderived chemotactic factor, originally identified as CC chemokine ligand 2 (CCL2; also known as MCP-1) (32). Following this observation, other chemokines able to recruit monocytes were detected in neoplastic tissues as products of either the tumor cells or host stromal elements (55). In addition to recruiting monocytes, these molecules play an important role in tumor progression by directly stimulating neoplastic growth, promoting inflammation, and inducing angiogenesis (58). Evidence supporting a pivotal role for chemokines, in addition to CCL2, in the recruitment of monocytes to neoplastic tissues includes a direct correlation 
between chemokine production and monocyte infiltration in mouse and human tumors (32).

Molecules other than chemokines can also promote monocyte recruitment. In particular, tumor-derived cytokines such as VEGF and M-CSF promote monocyte recruitment as well as macrophage survival and proliferation, and their expression correlates with tumor growth (59). Some of these factors, expressed in the tumor microenvironment, also inhibit the differentiation of monocytes into DCs by activating STAT3-dependent signaling (9), thereby impairing the induction of DC-induced antigen-specific immune responses (60).

Several lines of evidence suggest that some circulating MDSCs reach the tumor site and become part of the tumor stroma, indicating that, in addition to peripheral monocytes, CD $11 \mathrm{~b}^{+} \mathrm{Gr}-1^{+}$ MDSCs might also be precursors of F $4 / 80^{+}$TAMs. Indeed, it has been shown that Gr-1+ $1^{+}$cells isolated from the spleens of tumorbearing mice can reach the tumor and become $\mathrm{F} 4 / 80^{+} \mathrm{TAMs}$ characterized by increased STAT1 phosphorylation and constitutive expression of ARG1 and NOS2 $(13,27)$ (Figure 1). In tumor-bearing hosts, increased bioavailability of VEGF and release of soluble KIT ligand in the bone marrow are promoted through the high expression of MMP9 by splenic CD11 $\mathrm{b}^{+} \mathrm{Gr}^{+}$cells, which indirectly promote tumor vascularization and regulate the mobilization of more $\mathrm{CD} 11 \mathrm{~b}^{+} \mathrm{Gr}^{+}$cells. These $\mathrm{CD} 11 \mathrm{~b}^{+} \mathrm{Gr}-1^{+}$cells were also found to directly incorporate into the tumor endothelium (61), where they contribute to tumor growth and vascularization by producing MMP9 and differentiating into endothelial cells. Moreover, the concept of a shared differentiation pathway between circulating MDSCs and TAMs (Figure 1) is reinforced by the common molecular pathways (activated by IFN- $\gamma$ and IL-13) necessary for their immunosuppressive activity, as previously described (11).

TAMs preferentially localize to poorly vascularized regions of tumors $(62,63)$. This environment promotes the metabolic adaptation of TAMs to hypoxia through the activation of hypoxiainducible factor 1 (HIF-1) and HIF-2 (63). We recently have shown that HIF- $1 \alpha$ activated in TAMs by hypoxia influences the positioning and function of tumor cells, stromal cells, and TAMs by selectively upregulating their expression of CXC chemokine receptor 4 (CXCR4) (64). Moreover, HIF-1 activation can have a role in the induction of the CXCR4 ligand, CXC chemokine ligand 12 (CXCL12) (65), a chemokine involved in cancer metastasis (66). Together, these data suggest that oxygen availability has a role in guiding the microanatomical localization and function of TAMs. Moreover, hypoxia can also have important consequences on L-Arg metabolism in TAMs and thereby on the suppression of adaptive immunity, since it can induce NOS2 and ARG expression (in this case with a certain variability in terms of ARG1 and ARG2) in various cell types (67-69).

In addition to HIF- $1 \alpha$, analysis of the molecular basis of the TAM phenotype has identified NF-KB as the master regulator of TAM transcriptional programs, and some evidence suggests that modulation of NF-KB activity in these cells is an important mechanism by which their protumoral functions can be controlled (32).

Although in inflammatory leukocytes, in particular macrophages, NF- $\mathrm{kB}$ is an essential transcription factor guiding the inflammatory response, this factor is also recognized as a major effector of cancer cell proliferation and survival (70). In cancer, NF- $\mathrm{kB}$ induces more aggressive tumor phenotypes by promoting cells to grow independently of growth signals; by increasing their insensitivity to growth inhibition; by increasing their resis- tance to apoptotic signals; by immortalizing the cells; by enhancing angiogenesis; and by enhancing tissue invasion and metastasis (71). The constitutive NF- $\mathrm{kB}$ activation often observed in tumor cells might be promoted by either signals from the microenvironment, including cytokines, hypoxia, and ROS, or by genetic alterations (71). In particular, proinflammatory cytokines (e.g., IL-1 and TNF- $\alpha$ ) expressed by different subsets of tumor-infiltrating leukocytes (72) can activate NF-KB in cancer cells and contribute to their proliferation and survival (71). Strikingly, the proliferative role of TNF- $\alpha$ was recently confirmed in primary and in vitro-established human renal carcinoma cells (73). The peculiar ability of tumors to promote leukocyte recruitment largely relies on their constitutive expression of the genes that encode inflammatory chemokines, whose expression is controlled by NF-KB (74). These data underpin the central role of NF- $\mathrm{KB}$ in the functional crosstalk between tumors and the immune system and suggest a causal relationship between NF-KB-mediated inflammation and tumorigenesis (70).

Differences are emerging about the effects of NF- $\mathrm{KB}$ in cancer cells and TAMs. In contrast with cancer cells, in fact, TAMs from advanced tumors show defective NF- $\kappa \mathrm{B}$ activation in response to different proinflammatory signals $(55,75,76)$. This defective NF- $\kappa B$ activation in TAMs correlates with impaired expression of NF-KB-dependent inflammatory functions (e.g., the expression of cytotoxic mediators such as TNF- $\alpha$, IL-1, and IL-12) (32). These observations are in apparent contrast with a protumor function of inflammatory reactions observed in models of spontaneous or chemically induced carcinogenesis $(77,78)$. Although in these latter models, NF-кB inhibition resulted in tumor growth delay $(77,78)$, in tumors at a more advanced stage of progression, a therapeutic effect was achieved through the reactivation of NF- $\kappa \mathrm{B}$-dependent inflammation in the myeloid cell compartment $(75,79,80)$. This discrepancy might reflect a dynamic change in the tumor microenvironment during the transition from early neoplastic events to advanced tumor stages, which would result in progressive modulation of the NF-KB activity expressed by infiltrating inflammatory cells and progressive conversion of the TAMs from an M1 to an M2 macrophage phenotype. Importantly, restoration of NF-кB activity in TAMs from advanced tumors results in increased expression of inflammatory cytokines (e.g., TNF- $\alpha$ ) and is associated with a delay in tumor growth (75). So far, NF- $\mathrm{KB}$ pathways have been characterized, in part, in TAMs, and similar studies should be replicated in MDSCs.

\section{TAMs mediate an M2 macrophage-oriented persistent inflammation}

Characterization of the transcriptome of TAMs isolated from a mouse fibrosarcoma confirmed that these cells mainly have an M2 macrophage phenotype but also express IFN-inducible chemokines (a characteristic of M1 macrophages) (81). A similar mixture of gene profiles (mostly an M2 profile with M1 traits) was also recently found in mouse MDSCs (11). The mainly M2 macrophage-like phenotype of TAMs is associated with them having protumoral function. Evidence for this comes from a number of studies. First, pharmacological skewing of TAM polarization from an M2 macrophage-like phenotype to a full M1 macrophage phenotype sustains antitumor immunity $(79,82)$. Indeed, a combination of $\mathrm{CPG}$ oligodeoxynucleotides and an IL-10 receptor-specific antibody switched TAMs from an M2 to an M1 macrophage-like phenotype and triggered an innate response that was able to debulk large tumors within 16 hours (82). Second, 


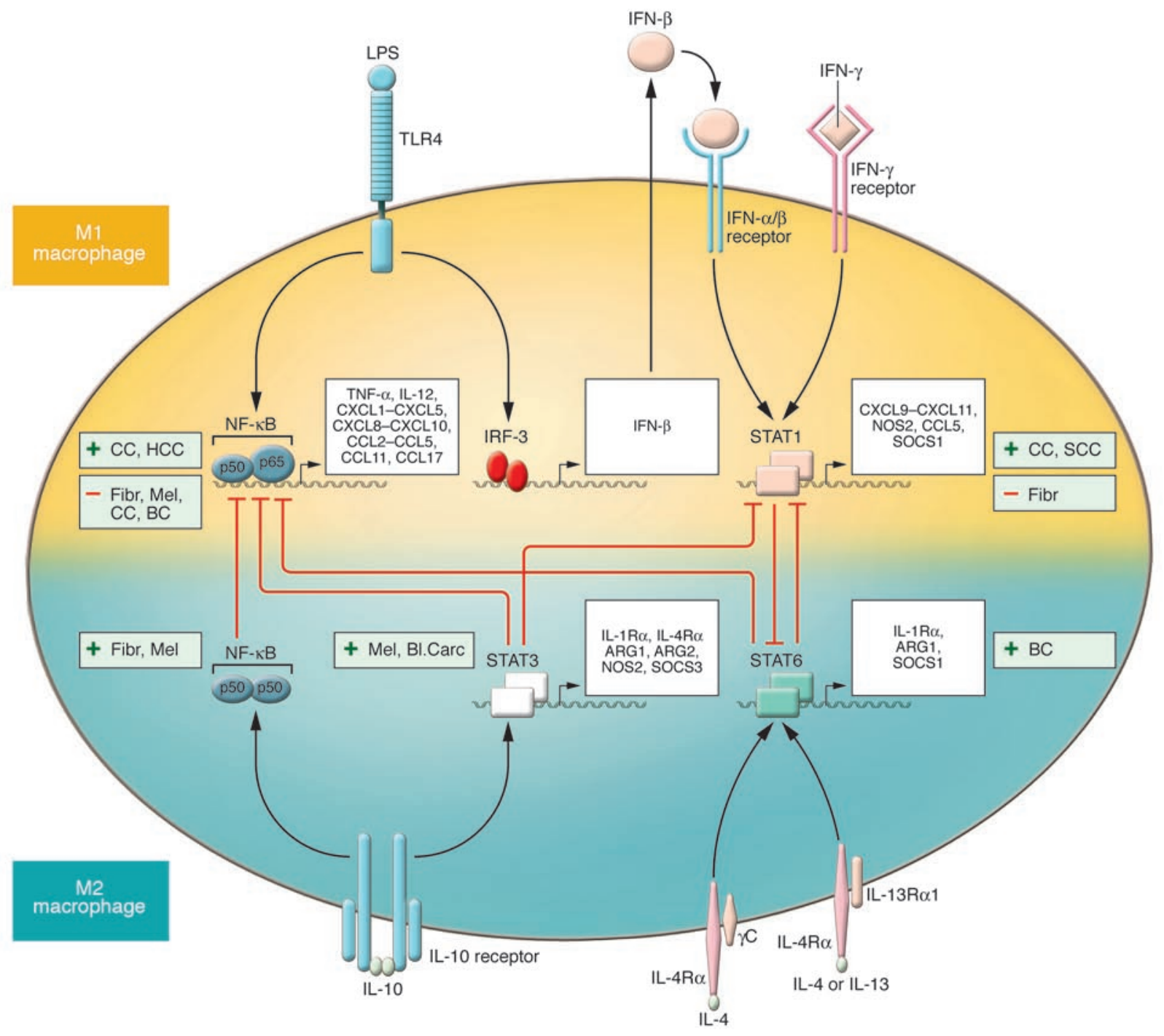

\section{Figure 3}

Molecular pathways of macrophage polarization and their role in tumor progression. The major pathways of macrophage polarization and current evidence linking their activation with either tumor progression (+) or regression $(-)$ are outlined. The overall view suggests that M2 macrophage-polarizing signals (such as IL-10, IL-4, and IL-13) are mainly associated with tumor progression. Contrasting evidence associates M1 macrophage-polarizing pathways (such as IFN- $\gamma$ and TLR ligation) with either tumor progression or regression. The crosstalk between the M1 and M2 macrophage-polarizing pathways, which results in reciprocal modulation, are also indicated. As shown, IL-10-mediated induction of the p50 NF-KB homodimer interferes with NF- $\mathrm{KB}$ activation and M1 macrophage-induced inflammation. The balance between activation of M1 macrophage-associated STAT1 and M2 macrophage-associated STAT3 and STAT6 finely regulates macrophage polarization and activity. A predominance of NF-кB and STAT1 activation results in M1 macrophage polarization, which promotes cytotoxic and inflammatory functions. In contrast, a predominance of STAT3 and STAT6 activation results in M2 macrophage polarization, which is associated with immune suppression and tumor progression. As discussed in the text, IL-23 might also contribute to the polarization decision as it activates different STATs, including STAT1 and STAT3, in TAMs, but direct evidence is missing. CC, colorectal carcinoma; HCC, hepatocarcinoma; Fibr, fibrosarcoma; Mel, melanoma; BC, breast carcinoma; SCC, squamous cell carcinoma; BI. Carc, bladder carcinoma.

recent results suggest that SRC homology 2 domain-containing inositol-5-phosphatase (SHIP) functions in vivo to repress skewing to an M2 macrophage-like phenotype. Peritoneal and alveolar macrophages isolated from Ship ${ }^{-/-}$mice constitutively express high levels of ARG1 and show impaired LPS-induced NO production. Consistent with this, transplanted tumors grow more rapidly in $\mathrm{Ship}^{-/-}$mice than in wild-type mice $(83,84)$. Third, a DNA vaccine against the M2 macrophage-associated molecule legumain, which is highly expressed by TAMs, induced a robust $\mathrm{CD}^{+} \mathrm{T}$ cell response against TAMs, reducing their density in tumor tissues and leading to the suppression of angiogenesis, tumor growth, and metastasis (85). Finally, we have recently demonstrated that TAMs are characterized by nuclear localization of the inhibitory p50 NF-кB homodimer, a phenotype associated with tumor progression and a lack of M1 macrophage-like function (75). Interestingly, the M2 macrophage-inducing signals PGE 2 , IL-10, and TGF- $\beta$ were shown to promote increased nuclear localization of the p50 NF-кB homodimer (75). Moreover, mice lacking expres- 
sion of p50 also lack expression of the M2 macrophage-polarizing cytokines IL-4, IL-5, and IL-13 (86), and in tumor-bearing mice lacking expression of p50, TAMs express cytokines characteristic of M1 macrophages, and splenocytes produce Th1 cytokines, both of which are associated with a delay in tumor growth (75).

All these findings together suggest that M2 macrophage-like inflammation fuels cancer progression and lead to the suggestion that NF- $\mathrm{KB}$ inhibition in TAMs is associated with M2 macrophage-like inflammatory functions. It is probable that, although full activation of NF- $\mathrm{KB}$ in macrophages resident in preneoplastic sites might exacerbate local M1 macrophage-like inflammation and favor tumorigenesis $(77,78,87)$, tumor growth results in progressive inhibition of NF- $\mathrm{KB}$ in infiltrating leukocytes, as observed in both myeloid $(75,88)$ and lymphoid $(89)$ cells from individuals with tumors, and in the progressive skewing to M2 macrophage-like inflammation. If so, the therapeutic efficacy of strategies targeting NF- $\mathrm{KB}$ for the treatment of cancers might be determined by both the tumor stage and polarization status of the infiltrating leukocytes.

\section{STATs in TAM and MDSC function}

A central role in the polarization of myeloid cell functions as well as in tumor progression and the altered immune response to cancer is emerging for selected members of the STAT family of transcription factors. In particular, STAT1, STAT3, and STAT6 have been shown to have a major role in transmitting polarizing signals to the nucleus (90) and to have distinct roles in macrophage polarization (Figure 3). STAT1 is activated in response to M1 macrophage-polarizing signals (e.g., IFN- $\gamma$ and LPS) whereas STAT3 and STAT 6 are selectively activated by M2 macrophage-polarizing cytokines (e.g., IL-10, IL-4, and IL-13) (91). Activation of specific STATs, central inducers of macrophage polarization programs, is expected to parallel either the antitumoral or protumoral role of M1 and M2 macrophage-mediated inflammation, respectively.

Original evidence indicates that STAT1 activation is essential for immune surveillance against tumors (92). In particular, mice deficient for either the IFN- $\gamma$ receptor (signaling through which activates STAT1; ref. 93) or STAT1 displayed enhanced resistance to the induction of tumors by methylcholanthrene (94). Over the years, the STAT1-mediated antitumoral effect has been confirmed in preclinical tumor models $(95,96)$. However, recent reports argue against this simple view and suggest that the IFN- $\gamma /$ STAT1 pathway might have a protumoral role, at least in certain tumors. For example, STAT1 was recently described as responsible for TAM-mediated suppressive activity and tumor progression, and it was shown that TAMs isolated from STAT1-deficient mice failed to suppress $T$ cell responses (13). In addition, in a mouse squamous cell carcinoma, STAT1 deficiency enhanced IL-12-mediated tumor regression by a $\mathrm{T}$ cell-dependent mechanism (97). In agreement with the role of STAT1 as the central mediator of the biological activities of IFN- $\gamma$, administration of neutralizing antibodies specific for IFN- $\gamma$ inhibited tumor growth in IL-12-treated Stat $1^{+/+}$mice (97). More recently, it has also been shown that activation of the $\mathrm{CD} 8^{+} \mathrm{T}$ cell suppressive activity of tumor-induced MDSCs requires the action of IFN- $\gamma$, though in combination with IL-13 (11). In line with this picture, mice lacking SOCS1, which are characterized by hyperactivation of STAT1, display spontaneous development of colorectal carcinomas (98), supporting the idea that persistent activation of STAT1dependent signaling might be associated with tumor progression. Interestingly, molecular analysis of the transcriptome of TAMs showed that these cells express high levels of IFN-inducible chemokines and STAT1 activity (81). Together, these results suggest that, along with a predominant expression of $\mathrm{M} 2$ macrophage-polarized functions in TAMs and MDSCs, the parallel activation of STAT1 in these cells might enhance immune dysfunctions, further favoring tumor progression. This contrasting evidence on the influence of STAT1 might be explained by differences among the tumor models investigated, the state of tumor progression, and the number and type of infiltrating leukocytes.

STAT3 and STAT6 activation are associated with M2 macrophage polarization $(32,91)$. It has been shown that STAT3 is constitutively activated in tumor cells (99) and in diverse tumor-infiltrating immune cells, including TAMs (80), leading to inhibition of proinflammatory cytokine and chemokine production and to the release of factors that suppress DC maturation. Ablating STAT3 in hematopoietic cells triggers an intrinsic immune surveillance system that inhibits tumor growth and metastasis and is associated with enhanced functional activity of DCs, T cells, NK cells, and neutrophils (80). STAT3/JAK2 activation in myeloid cells by tumor-derived factors can lead to the accumulation of CD $11 \mathrm{~b}^{+} \mathrm{Gr}-1^{+}$MDSCs, preventing their differentiation into mature DCs, whereas interfering with STAT3 signaling reverses these inhibitory effects $(100,101)$. TAMs from Stat $6^{-/}$tumor-bearing mice display an M1 macrophage phenotype, with low levels of expression of ARG1 and high levels of expression of NOS2, which promotes tumor cell death through the cytotoxic activity of the high levels of NO that are produced. As a result, these mice rejected spontaneous mammary carcinomas in an immune system-dependent manner $(20,102)$. Therefore, although current literature strongly suggests a crucial role for polarized inflammation in cancer progression, additional studies should clarify whether accumulating and contrasting evidence might be ascribed to specific microenvironmental conditions or related to tumor type and/or stage of disease.

The recent observation that the cytokine IL-23, a member of the IL-12 cytokine family, is expressed in human and mouse tumors has unveiled another potential player in TAM-dependent immunosuppression. In mouse tumor models, expression of the mRNA encoding the IL-23p19 subunit was increased in $\mathrm{CD}_{11} \mathrm{~b}^{+}$and CD $11 \mathrm{c}^{+}$cells (probably TAMs and DCs) present in tumor stroma. Similarly to IL-12, IL-23 promotes inflammatory responses, but the net effect of the cytokine is deleterious for antitumor immunity. IL-23, in fact, promotes upregulation of MMP9 and increases tumor angiogenesis but reduces $\mathrm{CD}^{+} \mathrm{T}$ cell infiltration (103). Importantly, genetic deletion studies and antibody-mediated neutralization of IL-23 have demonstrated a direct negative effect of the cytokine on tumor immune surveillance (103). Furthermore, IL-23 stimulation can activate STAT1, STAT3, STAT4, and STAT5 and lead to enhanced production of IL-6 (104); it therefore might have an important role in influencing the TAM transcriptome and function.

\section{For the future: therapeutic perspectives}

MDSCs and TAMs probably represent a continuum of a unique myeloid cell-differentiation program induced by tumor-derived factors to support an incessant influx of cells that aid tumor invasion of nearby tissues, stroma remodeling, and cell proliferation and that inhibit the innate and adaptive antitumor immune response. Targeting this dynamic process might offer interesting perspectives for new therapies for the treatment of cancer $(4,79)$. In applying novel approaches to relieving the immunosuppression 
induced by MDSCs and TAMs, one aspect must be considered: the relative contribution of MDSCs and TAMs to the overall impairment of antitumor $\mathrm{T}$ cell responses has not been clearly estimated. It is probable that inhibition of $\mathrm{CD}^{+} \mathrm{T}$ cell antitumor immunity by MDSCs and TAMs in tumor-bearing hosts might occur in different places, primarily the tumor site and the draining lymph nodes but also distant sites of the immune system. MDSCs and TAMs might also affect differently the subsets of circulating $\mathrm{CD}^{+}$ $\mathrm{T}$ cells in relation to the spread of malignant tumors in different patients. We think that combining protocols that interfere with MDSC- and/or TAM-mediated immune suppression with either cancer vaccination (active immunotherapy) or the adoptive transfer of ex vivo-expanded tumor-infiltrating $T$ cells (passive immunotherapy) might provide therapeutic benefit for the treatment of cancer. However, the benefit of such combination approaches is likely to differ in every patient according to the state of impairment of the antitumor immune response.

\section{Acknowledgments}

We thank Alberto Mantovani for his critical reading and constant suggestions. This work has been supported by grants from the Italian Ministry of Health, the Italian Foundation for Multiple Sclerosis (FISM), the Italian Association for Cancer Research (AIRC), and the European Community.

Address correspondence to: Vincenzo Bronte, Istituto Oncologico Veneto, Via Gattamelata 64, 35128 Padua, Italy. Phone: 39-0498215897; Fax: 39-049-8072854; E-mail: enzo.bronte@unipd.it.
1. Mocellin, S., et al. 2004. Part I: Vaccines for solid tumours. Lancet Oncol. 5:681-689.

2. Rosenberg, S.A., Yang, J.C., and Restifo, N.P. 2004 Cancer immunotherapy: moving beyond current vaccines. Nat. Med. 10:909-915.

3. Rabinovich, G.A., Gabrilovich, D., and Sotomayor, E.M. 2007. Immunosuppressive strategies that are mediated by tumor cells. Annu. Rev. Immunol. 25:267-296.

4. Serafini, P., Borrello, I., and Bronte, V. 2006 Myeloid suppressor cells in cancer: recruitment, phenotype, properties, and mechanisms of immune suppression. Semin. Cancer Biol. 16:53-65.

5. Maier, T., Holda, J.H., and Claman, H.N. 1989. Natural suppressor cells. Prog. Clin. Biol. Res. 288:235-244.

6. Seung, L.P., Rowley, D.A., Dubey, P., and Schreiber, H. 1995. Synergy between T-cell immunity and inhibition of paracrine stimulation causes tumor rejection. Proc. Natl. Acad. Sci. U. S. A. 92:6254-6258.

7. Pekarek, L.A., Starr, B.A., Toledano, A.Y., and Schreiber, H. 1995. Inhibition of tumor growth by elimination of granulocytes. J. Exp. Med. 181:435-440.

8. Kusmartsev, S., and Gabrilovich, D.I. 2006. Role of immature myeloid cells in mechanisms of immune evasion in cancer. Cancer Immunol. Immunother. 55:237-245

9. Gabrilovich, D. 2004. Mechanisms and functional significance of tumour-induced dendritic-cell defects. Nat. Rev. Immunol. 4:941-952.

10. Gabrilovich, D.I., et al. 2007. The terminology issue for myeloid-derived suppressor cells. Cancer Res. 67:425; author reply 426.

11. Gallina, G., et al. 2006. Tumors induce a subset of inflammatory monocytes with immunosuppressive activity on CD8 T cells. J. Clin. Invest. 116:2777-2790. doi:10.1172/JCI28828.

12. Van Ginderachter, J.A., et al. 2006. Peroxisome proliferator-activated receptor gamma (PPARga$\mathrm{mma}$ ) ligands reverse CTL suppression by alternatively activated (M2) macrophages in cancer. Blood. 108:525-535.

13. Kusmartsev, S., and Gabrilovich, D.I. 2005. STAT1 signaling regulates tumor-associated macrophagemediated T cell deletion. J. Immunol. 174:4880-4891.

14. Tsuda, Y., et al. 2004. Three different neutrophil subsets exhibited in mice with different susceptibilities to infection by methicillin-resistant Staphylococcus aureus. Immunity. 21:215-226.

15. Zea, A.H., et al. 2005. Arginase-producing myeloid suppressor cells in renal cell carcinoma patients: a mechanism of tumor evasion. Cancer Res. 65:3044-3048.

16. Schmielau, J., and Finn, O.J. 2001. Activated granulocytes and granulocyte-derived hydrogen peroxide are the underlying mechanism of suppression of $\mathrm{t}$-cell function in advanced cancer patients. Cancer Res. 61:4756-4760.

17. Bronte, V., et al. 1999. Unopposed production of granulocyte-macrophage colony-stimulating fac- tor by tumors inhibits CD8+ T cell responses by dysregulating antigen-presenting cell maturation. J. Immunol. 162:5728-5737.

18. Melani, C., Chiodoni, C., Forni, G., and Colombo, M.P. 2003. Myeloid cell expansion elicited by the progression of spontaneous mammary carcinomas in c-erbB-2 transgenic BALB/c mice suppresses immune reactivity. Blood. 102:2138-2145.

19. Kusmartsev, S., Nefedova, Y., Yoder, D., and Gabrilovich, D.I. 2004. Antigen-specific inhibition of CD8+ T cell response by immature myeloid cells in cancer is mediated by reactive oxygen species. J. Immunol. 172:989-999.

20. Sinha, P., Clements, V.K., and Ostrand-Rosenberg, S. 2005. Reduction of myeloid-derived suppressor cells and induction of M1 macrophages facilitate the rejection of established metastatic disease. J. Immunol. 174:636-645.

21. Terabe, M., et al. 2000. NKT cell-mediated repression of tumor immunosurveillance by IL-13 and the IL-4R-STAT6 pathway. Nat. Immunol. 1:515-520.

22. Terabe, M., et al. 2003. Transforming growth factor-\{beta\} production and myeloid cells are an effector mechanism through which CD1d-restricted $\mathrm{T}$ cells block cytotoxic T lymphocyte-mediated tumor immunosurveillance: abrogation prevents tumor recurrence. J. Exp. Med. 198:1741-1752.

23. Bronte, V., and Zanovello, P. 2005. Regulation of immune responses by L-arginine metabolism [review]. Nat. Rev. Immunol. 5:641-654.

24. Gabrilovich, D.I., Velders, M.P., Sotomayor, E.M., and Kast, W.M. 2001. Mechanism of immune dysfunction in cancer mediated by immature gr-1(+) myeloid cells. J. Immunol. 166:5398-5406.

25. Otsuji, M., et al. 1996. Oxidative stress by tumorderived macrophages suppresses the expression of CD3 zeta chain of T-cell receptor complex and antigen-specific T-cell responses. Proc. Natl. Acad. Sci. U. S. A. 93:13119-13124.

26. Huang, B., et al. 2006. Gr-1+CD115+ immature myeloid suppressor cells mediate the development of tumor-induced $\mathrm{T}$ regulatory cells and T-cell anergy in tumor-bearing host. Cancer Res. 66:1123-1131.

27. Kusmartsev, S., Nagaraj, S., and Gabrilovich, D.I. 2005. Tumor-associated CD8+ T cell tolerance induced by bone marrow-derived immature myeloid cells. J. Immunol. 175:4583-4592.

28. Bronte, V., et al. 1998. Apoptotic death of CD8+ $T$ lymphocytes after immunization: induction of a suppressive population of Mac-1+/Gr-1+ cells. J. Immunol. 161:5313-5320.

29. Serafini, P., et al. 2006. Phosphodiesterase-5 inhibition augments endogenous antitumor immunity by reducing myeloid-derived suppressor cell function. J. Exp. Med. 203:2691-2702.

30. Yang, R., et al. 2006. CD80 in immune suppression by mouse ovarian carcinoma-associated $\mathrm{Gr}$ 1+CD11b+ myeloid cells. Cancer Res. 66:6807-6815.

31. Gordon, S. 2003. Alternative activation of macrophages. Nat. Rev. Immunol. 3:23-35.

32. Mantovani, A., et al. 2002. Macrophage polarization: tumor-associated macrophages as a paradigm for polarized M2 mononuclear phagocytes. Trends Immunol. 23:549-555.

33. Mantovani, A., et al. 2004. The chemokine system in diverse forms of macrophage activation and polarization. Trends Immunol. 25:677-686.

34. Ganster, R.W., Taylor, B.S., Shao, L., and Geller, D.A. 2001. Complex regulation of human inducible nitric oxide synthase gene transcription by Stat 1 and NF-kappa B. Proc. Natl. Acad. Sci. U. S. A. 98:8638-8643.

35. Kleinert, H., Schwarz, P.M., and Forstermann, U. 2003. Regulation of the expression of inducible nitric oxide synthase. Biol. Chem. 384:1343-1364.

36. Melillo, G., et al. 1995. A hypoxia-responsive element mediates a novel pathway of activation of the inducible nitric oxide synthase promoter. J. Exp. Med. 182:1683-1693.

37. Boutard, V., et al. 1995. Transforming growth factorbeta stimulates arginase activity in macrophages. Implications for the regulation of macrophage cytotoxicity. J. Immunol. 155:2077-2084.

38. Morrison, A.C., and Correll, P.H. 2002. Activation of the stem cell-derived tyrosine kinase/RON receptor tyrosine kinase by macrophage-stimulating protein results in the induction of arginase activity in murine peritoneal macrophages. J. Immunol. 168:853-860.

39. Jost, M.M., et al. 2003. Divergent effects of GMCSF and TGFbeta1 on bone marrow-derived macrophage arginase- 1 activity, MCP- 1 expression, and matrix metalloproteinase-12: a potential role during arteriogenesis. FASEB J. 17:2281-2283.

40. Munder, M., Eichmann, K., and Modolell, M. 1998. Alternative metabolic states in murine macrophages reflected by the nitric oxide synthase/arginase balance: competitive regulation by CD4+ T cells correlates with Th1/Th2 phenotype. J. Immunol. 160:5347-5354.

41. Raes, G., et al. 2005. Arginase-1 and Ym1 are markers for murine, but not human, alternatively activated myeloid cells. J. Immunol. 174:6561; author reply 6561-6562.

42. Munder, M., et al. 2006. Suppression of T cell functions by human granulocyte arginase. Blood. 108:1627-1634.

43. Rodriguez, P.C., and Ochoa, A.C. 2006. T cell dysfunction in cancer: role of myeloid cells and tumor cells regulating amino acid availability and oxidative stress. Semin. Cancer Biol. 16:66-72.

44. Bronte, V., et al. 2003. IL-4-induced arginase 1 suppresses alloreactive $\mathrm{T}$ cells in tumor-bearing mice. J. Immunol. 170:270-278.

45. Mazzoni, A., et al. 2002. Myeloid suppressor lines inhibit $\mathrm{T}$ cell responses by an NO-dependent mechanism. J. Immunol. 168:689-695.

46. Apolloni, E., et al. 2000. Immortalized myeloid sup- 
pressor cells trigger apoptosis in antigen-activated T lymphocytes. J. Immunol. 165:6723-6730.

47. Rodriguez, P.C., et al. 2004. Arginase I production in the tumor microenvironment by mature myeloid cells inhibits T-cell receptor expression and antigen-specific T-cell responses. Cancer Res. 64:5839-5849.

48. Rodriguez, P.C., Quiceno, D.G., and Ochoa, A.C. 2006. L-Arginine availability regulates T lymphocyte cell cycle progression. Blood. 109:1568-1573.

49. Fallarino, F., et al. 2006. The combined effects of tryptophan starvation and tryptophan catabolites down-regulate $\mathrm{T}$ cell receptor zeta-chain and induce a regulatory phenotype in naive $\mathrm{T}$ cells. J. Immunol. 176:6752-6761.

50. Munn, D.H., et al. 2005. GCN2 kinase in T cells mediates proliferative arrest and anergy induction in response to indoleamine 2,3-dioxygenase. Immunity. 22:633-642.

51. Sinha, P., Clements, V.K., and Ostrand-Rosenberg, S. 2005. Interleukin-13-regulated M2 macrophages in combination with myeloid suppressor cells block immune surveillance against metastasis. Cancer Res. 65:11743-11751.

52. Ezernitchi, A.V., et al. 2006. TCR zeta down-regulation under chronic inflammation is mediated by myeloid suppressor cells differentially distributed between various lymphatic organs. J. Immunol. 177:4763-4772.

53. Baniyash, M. 2004. TCR zeta-chain downregulation: curtailing an excessive inflammatory immune response. Nat. Rev. Immunol. 4:675-687.

54. Bronte, V., et al. 2005. Boosting antitumor responses of $\mathrm{T}$ lymphocytes infiltrating human prostate cancers. J. Exp. Med. 201:1257-1268.

55. Sica, A., Schioppa, T., Mantovani, A., and Allavena, P. 2006. Tumour-associated macrophages are a distinct M2 polarised population promoting tumour progression: potential targets of anti-cancer therapy. Eur. J. Cancer. 42:717-727.

56. Bingle, L., Brown, N.J., and Lewis, C.E. 2002. The role of tumour-associated macrophages in tumour progression: implications for new anticancer therapies. J. Pathol. 196:254-265.

57. Mantovani, A., et al. 1992. The origin and function of tumor-associated macrophages. Immunol. Today. 13:265-270.

58. Balkwill, F. 2003. Chemokine biology in cancer. Semin. Immunol. 15:49-55.

59. Pollard, J.W. 2004. Tumour-educated macrophages promote tumour progression and metastasis. Nat. Rev. Cancer. 4:71-78.

60. Mellman, I., and Steinman, R.M. 2001. Dendritic cells: specialized and regulated antigen processing machines. Cell. 106:255-258.

61. Yang, L., et al. 2004. Expansion of myeloid immune suppressor $\mathrm{Gr}+\mathrm{CD} 11 \mathrm{~b}+$ cells in tumor-bearing host directly promotes tumor angiogenesis. Cancer Cell. 6:409-421.

62. Lewis, C.E., and Pollard, J.W. 2006. Distinct role of macrophages in different tumor microenvironments. Cancer Res. 66:605-612.

63. Leek, R.D., et al. 2002. Relation of hypoxia-inducible factor-2 alpha (HIF-2 alpha) expression in tumor-infiltrative macrophages to tumor angiogenesis and the oxidative thymidine phosphorylase pathway in human breast cancer. Cancer Res. 62:1326-1329.

64. Schioppa, T., et al. 2003. Regulation of the chemokine receptor CXCR4 by hypoxia. J. Exp. Med. 198:1391-1402.

65. Ceradini, D.J., et al. 2004. Progenitor cell trafficking is regulated by hypoxic gradients through HIF-1 induction of SDF-1. Nat. Med. 10:858-864.

66. Muller, A., et al. 2001. Involvement of chemokine receptors in breast cancer metastasis. Nature. 410:50-56.

67. Albina, J.E., et al. 2005. Macrophage arginase regu- lation by CCAAT/enhancer-binding protein beta. Shock. 23:168-172.

68. Morris, S.M., Jr. 2002. Regulation of enzymes of the urea cycle and arginine metabolism. Annu. Rev. Nutr. 22:87-105.

69. Semenza, G.L. 2003. Targeting HIF-1 for cancer therapy. Nat. Rev. Cancer. 3:721-732.

70. Karin, M., and Greten, F.R. 2005. NF-kappaB: linking inflammation and immunity to cancer development and progression. Nat. Rev. Immunol. 5:749-759.

71. Karin, M., and Lin, A. 2002. NF-kappaB at the crossroads of life and death. Nat. Immunol. 3:221-227.

72. Balkwill, F., Charles, K.A., and Mantovani, A. 2005. Smoldering and polarized inflammation in the initiation and promotion of malignant disease. Cancer Cell. 7:211-217

73. Falkensammer, C., et al. 2006. IL-4 inhibits the TNF-alpha induced proliferation of renal cell carcinoma (RCC) and cooperates with TNF-alpha to induce apoptotic and cytokine responses by RCC: implications for antitumor immune responses. Cancer Immunol. Immunother. 55:1228-1237.

74. Richmond, A. 2002. Nf-kappa B, chemokine gene transcription and tumour growth. Nat. Rev. Immunol. 2:664-674.

75. Saccani, A., et al. 2006. p50 nuclear factor-\{kappa\}B overexpression in tumor-associated macrophages inhibits M1 inflammatory responses and antitumor resistance. Cancer Res. 66:11432-11440.

76. Sica, A., et al. 2000. Autocrine production of IL-10 mediates defective IL-12 production and NF-kappa $B$ activation in tumor-associated macrophages. J. Immunol. 164:762-767.

77. Greten, F.R., and Karin, M. 2004. The IKK/NF-kap$\mathrm{paB}$ activation pathway-a target for prevention and treatment of cancer. Cancer Lett. 206:193-199.

78. Pikarsky, E., et al. 2004. NF-kappaB functions as a tumour promoter in inflammation-associated cancer. Nature. 431:461-466.

79. Colombo, M.P., and Mantovani, A. 2005. Targeting myelomonocytic cells to revert inflammation-dependent cancer promotion. Cancer Res. 65:9113-9116.

80. Kortylewski, M., et al. 2005. Inhibiting Stat3 signaling in the hematopoietic system elicits multicomponent antitumor immunity. Nat. Med. 11:1314-1321.

81. Biswas, S.K., et al. 2006. A distinct and unique transcriptional program expressed by tumor-associated macrophages (defective NF-kappaB and enhanced IRF-3/STAT1 activation). Blood. 107:2112-2122.

82. Guiducci, C., et al. 2005. Redirecting in vivo elicited tumor infiltrating macrophages and dendritic cells towards tumor rejection. Cancer Res. 65:3437-3446.

83. Rauh, M.J., et al. 2005. SHIP represses the generation of alternatively activated macrophages. Immunity. 23:361-374.

84. Mantovani, A., Sica, A., and Locati, M. 2005. Macrophage polarization comes of age. Immunity. 23:344-346.

85. Luo, Y., et al. 2006. Targeting tumor-associated macrophages as a novel strategy against breast cancer. J. Clin. Invest. 116:2132-2141. doi: 10.1172/ JCI27648.

86. Das, J., et al. 2001. A critical role for NF-kappa B in GATA 3 expression and TH 2 differentiation in allergic airway inflammation. Nat. Immunol. 2:45-50.

87. Moore, R.J., et al. 1999. Mice deficient in tumor necrosis factor-alpha are resistant to skin carcinogenesis. Nat. Med. 5:828-831.

88. Sica, A., et al. 2000. Defective expression of the monocyte chemotactic protein-1 receptor CCR2 in macrophages associated with human ovarian carcinoma. J. Immunol. 164:733-738.

89. Ghosh, P., et al. 1994. Alterations in NF kappa B/ Rel family proteins in splenic T-cells from tumorbearing mice and reversal following therapy. Cancer Res. 54:2969-2972.

90. Yoshimura, A. 2006. Signal transduction of inflam- matory cytokines and tumor development. Cancer Sci. 97:439-447.

91. O'Shea, J.J., Pesu, M., Borie, D.C., and Changelian, P.S. 2004. A new modality for immunosuppression: targeting the JAK/STAT pathway. Nat. Rev. Drug Discov. 3:555-564.

92. Dunn, G.P., Koebel, C.M., and Schreiber, R.D. 2006. Interferons, immunity and cancer immunoediting. Nat. Rev. Immunol. 6:836-848.

93. Bach, E.A., Aguet, M., and Schreiber, R.D. 1997. The IFN gamma receptor: a paradigm for cytokine receptor signaling. Annu. Rev. Immunol. 15:563-591.

94. Kaplan, D.H., et al. 1998. Demonstration of an interferon gamma-dependent tumor surveillance system in immunocompetent mice. Proc. Natl. Acad. Sci. U. S. A. 95:7556-7561.

95. Lesinski, G.B., et al. 2003. The antitumor effects of IFN-alpha are abrogated in a STAT1-deficient mouse. J. Clin. Invest. 112:170-180. doi: 10.1172/ JCI200316603.

96. Zhou, Y., Wang, S., Gobl, A., and Oberg, K. 2001. Interferon alpha induction of Stat 1 and Stat 2 and their prognostic significance in carcinoid tumors. Oncology. 60:330-338.

97. Torrero, M.N., et al. 2006. Stat 1 deficiency in the host enhances interleukin-12-mediated tumor regression. Cancer Res. 66:4461-4467.

98. Hanada, T., et al. 2006. IFNgamma-dependent, spontaneous development of colorectal carcinomas in SOCS1-deficient mice. J. Exp. Med. 203:1391-1397.

99. Wang, T., et al. 2004. Regulation of the innate and adaptive immune responses by Stat-3 signaling in tumor cells. Nat. Med. 10:48-54.

100.Nefedova, Y., et al. 2004. Hyperactivation of STAT3 is involved in abnormal differentiation of dendritic cells in cancer. J. Immunol. 172:464-474.

101. Nefedova, Y., et al. 2005. Activation of dendritic cells via inhibition of Jak2/STAT3 signaling. J. Immunol. 175:4338-4346.

102. Ostrand-Rosenberg, S., et al. 2002. Resistance to metastatic disease in STAT6-deficient mice requires hemopoietic and nonhemopoietic cells and is IFNgamma dependent. J. Immunol. 169:5796-5804.

103.Langowski, J.L., et al. 2006. IL-23 promotes tumour incidence and growth. Nature. 442:461-465.

104.Watford, W.T., et al. 2004. Signaling by IL-12 and IL-23 and the immunoregulatory roles of STAT4. Immunol. Rev. 202:139-156.

105.Serafini, P., et al. 2004. High-dose granulocytemacrophage colony-stimulating factor-producing vaccines impair the immune response through the recruitment of myeloid suppressor cells. Cancer Res. 64:6337-6343.

106.Condeelis, J., and Pollard, J.W. 2006. Macrophages: obligate partners for tumor cell migration, invasion, and metastasis. Cell. 124:263-266.

107. Talks, K.L., et al. 2000. The expression and distribution of the hypoxia-inducible factors HIF-1alpha and HIF-2alpha in normal human tissues, cancers, and tumor-associated macrophages. Am. J. Pathol. 157:411-421.

108. Bolat, F., et al. 2006. Microvessel density, VEGF expression, and tumor-associated macrophages in breast tumors: correlations with prognostic parameters. J. Exp. Clin. Cancer Res. 25:365-372.

109.Ueno, T., et al. 2000. Significance of macrophage chemoattractant protein-1 in macrophage recruitment, angiogenesis, and survival in human breast cancer. Clin. Cancer Res. 6:3282-3289.

110.Kataki, A., et al. 2002. Tumor infiltrating lymphocytes and macrophages have a potential dual role in lung cancer by supporting both host-defense and tumor progression. J. Lab. Clin. Med. 140:320-328.

111. O'Sullivan, C., Lewis, C.E., Harris, A.L., and McGee, J.O. 1993. Secretion of epidermal growth factor by macrophages associated with breast carcinoma. Lancet. 342:148-149.

112.van Kempen, L.C., and Coussens, L.M. 2002. MMP9 
potentiates pulmonary metastasis formation. Cancer Cell. 2:251-252.

113. Curiel, T.J., et al. 2004. Specific recruitment of regulatory $\mathrm{T}$ cells in ovarian carcinoma fosters immune privilege and predicts reduced survival. Nat. Med. 10:942-949.

114. Schutyser, E., et al. 2002. Identification of biologically active chemokine isoforms from ascitic fluid and elevated levels of CCL18/pulmonary and activation-regulated chemokine in ovarian carcinoma. J. Biol. Chem. 277:24584-24593.

115. Bronte, V., et al. 2000. Identification of a CD11b+/Gr$1+/ \mathrm{CD} 31+$ myeloid progenitor capable of activating or suppressing CD8+ T cells. Blood. 96:3838-3846.

116.Saio, M., Radoja, S., Marino, M., and Frey, A.B. 2001. Tumor-infiltrating macrophages induce apoptosis in activated CD8(+) T cells by a mechanism requiring cell contact and mediated by both the cell-associated form of TNF and nitric oxide.
J. Immunol. 167:5583-5593.

117.Young, M.R., et al. 1996. Suppression of T cell proliferation by tumor-induced granulocyte-macrophage progenitor cells producing transforming growth factor-beta and nitric oxide. J. Immunol. 156:1916-1922.

118.Liu, Y., et al. 2003. Nitric oxide-independent CTL suppression during tumor progression: association with arginase-producing (M2) myeloid cells. J. Immunol. 170:5064-5074.

119. Mencacci, A., et al. 2002. CD80+Gr-1+ myeloid cells inhibit development of antifungal Th1 immunity in mice with candidiasis. J. Immunol. 169:3180-3190.

120.Goni, O., Alcaide, P., and Fresno, M. 2002. Immunosuppression during acute Trypanosoma cruzi infection: involvement of Ly6G $(\mathrm{Gr} 1(+)) \mathrm{CD} 11 \mathrm{~b}(+)$ immature myeloid suppressor cells. Int. Immunol. 14:1125-1134.

121.Marshall, M.A., et al. 2001. Mice infected with
Schistosoma mansoni develop a novel non-T-lymphocyte suppressor population which inhibits virus-specific CTL induction via a soluble factor. Microbes Infect. 3:1051-1061.

122.Brys, L., et al. 2005. Reactive oxygen species and $12 / 15$-lipoxygenase contribute to the antiproliferative capacity of alternatively activated myeloid cells elicited during helminth infection. J. Immunol. 174:6095-6104.

123. Terrazas, L.I., et al. 2001. The schistosome oligosaccharide lacto-N-neotetraose expands Gr1(+) cells that secrete anti-inflammatory cytokines and inhibit proliferation of naive CD4(+) cells: a potential mechanism for immune polarization in helminth infections. J. Immunol. 167:5294-5303.

124.Giordanengo, L., et al. 2002. Cruzipain, a major Trypanosoma cruzi antigen, conditions the host immune response in favor of parasite. Eur. J. Immunol. 32:1003-1011. 